\title{
Genetik und Diagnostik des primären Lymphödems
}

\section{Genetics and diagnosis of primary lymphedema}

Autoren

Charlotte Kemper ${ }^{1}$, Magdalena Danyel ${ }^{1}$, Claus-Eric Ott ${ }^{1}$, René Hägerling ${ }^{1,2}$

Institute

1 Institut für Medizinische Genetik und Humangenetik, Charité - Universitätsmedizin Berlin

2 BIH Center for Regenerative Therapies, Berlin

Schlüsselwörter

Lymphödem, Molekulardiagnostik, lymphovaskuläre

Erkrankungen, syndromale Erkrankungen, Genetik

Key words

lymphoedema, molecular diagnostics, lymphovascular

diseases, syndromes, genetics

online publiziert 04.03 .2021

Bibliografie

Phlebologie 2021; 50: 105-114

DOI $10.1055 / a-1383-5360$

ISSN 0939-978X

(C) 2021. Thieme. All rights reserved.

Georg Thieme Verlag KG, Rüdigerstraße 14,

70469 Stuttgart, Germany

Korrespondenzadresse

Dr. rer. nat. Dr. med. René Hägerling

Charité-Universitätsmedizin Berlin

Institut für Medizinische Genetik und Humangenetik

Arbeitsgruppe „Lymphovaskuläre Medizin und translationale

3D-Histopathologie“, Deutschland

Tel.: +49/30/450569119

Rene.Haegerling@charite.de

\section{ZUSAMMENFASSUNG}

Einleitung Das primäre Lymphödem ist eine genetisch bedingte, angeborene Erkrankung, die durch einen unzureichenden Abtransport von Lymphflüssigkeit aufgrund einer Fehlbildung oder Fehlfunktion des Lymphgefäßsystems entsteht. Dabei tragen periphere und systemische Manifestationen zum letztendlichen Phänotyp bei. Neben peripheren Manifestationen des primären Lymphödems, v. a. Schwellungen der unteren Extremität, können auch systemische Manifestationen, wie z. B. Aszites, intestinale und pleurale Lymphangiektasien, Chylothorax, Pleura- und Perikarderguss oder auch der Hydrops fetalis, auftreten. In Abhängigkeit vom ursächlichen Gen und der zugrunde liegenden genetischen Veränderung unterscheiden sich sowohl die klinischen Manifestationen als auch der Ausprägungsgrad des Lymphödems.
Klassifikation Die Krankheitsbilder, die mit einem primären Lymphödem einhergehen, lassen sich in 5 Kategorien aufteilen: (1) Erkrankungen, die mit einer segmentalen Wachstumsstörung assoziiert sind und auf einem somatischen Mosaik beruhen, (2) syndromale Erkrankungen, (3) Erkrankungen, bei denen das primäre Lymphödem eine systemische Beteiligung aufweist, (4) kongenitale Krankheitsbilder und (5) nach dem ersten Lebensjahr auftretende (Late Onset) Krankheitsbilder. Genetische Diagnostik Basierend auf der Klinik des Patienten und der Zuordnung zu einer der 5 Kategorien kann eine zielgerichtete genetische Diagnostik erfolgen, zunächst beginnend mit einer konventionellen zytogenetischen Untersuchung (Chromosomenanalyse) sowie einer molekularzytogenetischen Methode (Array-CGH). Anschließend kann eine molekulargenetische Untersuchung im Rahmen von Einzelgenanalysen, Panel-Untersuchungen oder Exom- sowie Ganzgenomsequenzierung durchgeführt werden, durch die genetische Varianten oder Mutationen aufgedeckt werden können, die als kausal für die Symptomatik identifiziert werden können.

Fazit Betroffene eines primären Lymphödems profitieren von einer gezielten genetischen Diagnostik, da die verschiedenen Krankheitsbilder meistens nur durch die Detektion einer assoziierten genetischen Veränderung diagnostiziert werden können und somit eine Aussage über Vererbung und Wiederholungsrisiko möglich ist.

\section{ABSTRACT}

Introduction Primary lymphedema is a genetic, congenital disorder that results from impaired lymphatic drainage due to malformation or dysfunction of the lymphatic system. In addition to the peripheral manifestation of primary lymphedema, which often presents as edema of the extremities, particularly the lower extremity, systemic involvements such as hydrops fetalis, lymphangiectasia, or effusions of the serous cavities may also occur. The clinical presentation depends on the disease and its associated genes.

Classification The diseases associated with primary lymphedema can be divided into five categories: (1) Diseases associated with overgrowth which are based on somatic mosaicism, (2) syndromic diseases, (3) diseases in which primary lymphedema has systemic involvement, (4) congenital diseases, and (5) diseases occurring after the first year of life. Genetic diagnostic Depending on the patients' phenotype and assignment of appropriate category, targeted genetic diagnostic can be performed. Initially, starting with conven- 
tional cytogenetic testing (chromosome anaylsis) as well as molecular cytogenetic methods such as array-CGH diagnostic. Afterwards, molecular genetic testing can be performed in the context of single gene analysis, panel analysis and whole exome or whole genome sequencing, which can reveal genetic variants or mutations, that can be identified as causal for the symptoms.
Conclusion Affected individuals with primary lymphoedema benefit from genetic diagnostics because the different types of diseases can mostly only be diagnosed by the detection of an associated genetic alteration and thus a statement about heredity and recurrence risk can be made.

\section{Einleitung}

\subsection{Grundlagen des primären Lymphödems}

Das Lymphödem entsteht durch ein Missverhältnis zwischen der mikrovaskulären Filtration und dem Abtransport von Lymphflüssigkeit bzw. Lymphe, die sich u. a. aus proteinreicher Flüssigkeit und Immunzellen zusammensetzt. Durch eine verringerte Transportkapazität der Lymphgefäße kommt es dabei zu einer Flüssigkeitseinlagerung im Interstitium [1]. Aus diesem tritt die Lymphflüssigkeit, deren genaue Zusammensetzung je nach Herkunftsort variieren kann, zunächst durch kleinste, blind beginnende initiale Lymphgefäße in das Lymphgefäßsystem ein. Die initialen Lymphgefäße münden in Präkollektoren und im weiteren Verlauf in größerlumige Kollektoren. Während die Präkollektoren durch einzelne Zellen glatter Muskulatur gekennzeichnet sind, besitzen die Kollektoren und größeren Lymphstämme eine Schicht glatter Muskulatur. Klappen in den Präkollektoren und Kollektoren verhindern einen Rückfluss der Flüssigkeit der Gravitation folgend und ermöglichen den unidirektionalen Fluss der Lymphe. Durch das Zusammenwirken der Klappen und der Kontraktion glatter Muskulatur verfügt das Lymphgefäßsystem somit über einen eigenen Transportmechanismus. Dieser wird durch die Bewegungen der umgebenen Skelettmuskulatur und Gefäße unterstützt [2, 3].

Beim Lymphödem, das also durch eine Beeinträchtigung des Lymphgefäßsystems entsteht, unterscheidet man 2 Typen: Während das sekundäre Lymphödem Folge einer Vorerkrankung, eines Traumas oder einer iatrogen bedingten Schädigung ist, beruht das primäre Lymphödem auf einer angeborenen, in der Regel genetisch bedingten Fehlbildung der Lymphgefäße oder -klappen $[3,4]$.

\subsection{Klinische Manifestationen}

In Abhängigkeit vom assoziierten Gen und der zugrunde liegenden genetischen Veränderung unterscheiden sich sowohl die klinischen Manifestationen als auch der Ausprägungsgrad des Lymphödems [5]. Dabei tragen periphere und systemische Manifestationen zum letztendlichen Phänotyp bei. Periphere Manifestationen des primären Lymphödems sind Schwellungen im Bereich des Gesichts, des Stamms, der Genitalien oder der Extremitäten, wobei die untere Extremität am häufigsten betroffen ist. Zu den systemischen Manifestationen zählen Aszites, intestinale und pleurale Lymphangiektasien, Chylothorax, Pleura- und Perikarderguss, aber auch der Hydrops fetalis [5, 6]. Diesem generalisierten Ödem des Fötus mit pathologisch vermehrter Flüssigkeitsansammlung in fetalen serösen Körperhöhlen und Weichteilen liegt ein Ungleichgewicht der fetalen Flüssigkeitsbewegung zwischen dem Gefäßsystem und dem interstitiellen Raum zugrunde. Es handelt sich jedoch nicht um ein eigenständiges Krankheitsbild, sondern um ein Symptom oder Endstadium einer Vielzahl verschiedener Erkrankungen. Zu den Ursachen des ImmunHydrops fetalis zählen insbesondere maternofetale Immunreaktionen wie die Rhesus-Inkompatibilität oder Myasthenie. Häufiger jedoch liegt ein Non-Immun-Hydrops fetalis (NIHF) vor. Dieser beruht unter anderem auf hämatologischen Erkrankungen wie der genetisch bedingten Alpha-Thalassämie, kardiovaskulären oder anderen organischen Ursachen, Infektionen oder Stoffwechselstörungen, und kann idiopathisch, multifaktoriell oder im Rahmen eines genetisch bedingten Syndroms auf Basis größerer struktureller chromosomaler Veränderungen oder monogen, also auf Basis von Punktmutationen in einzelnen Genen, auftreten [7-9]. Insbesondere bei den monogen bedingten Störungen der embryonalen Lymphangiogenese kann der Hydrops fetalis Erstmanifestation des primären Lymphödems sein. Allerdings muss er nicht zwingend durch ein primäres Lymphödem begründet sein, sondern kann auch bei nicht lymphovaskulären Erkrankungen auftreten [5, 7].

\section{Klassifikation des primären Lymphödems}

Da die ursprüngliche Klassifikation in kongenitales Lymphödem, Lymphoedema praecox (Manifestation in der Pubertät) und Lymphoedema tarda (Manifestation nach dem 35. Lebensjahr) zu vereinfacht ist und den Phänotyp nicht mit einbezieht, haben Connell et al. 2010 einen Diagnostikalgorithmus entwickelt, um die verschiedenen Krankheitsbilder des primären Lymphödems anhand klinischer und genetischer Kriterien zu klassifizieren [4, 10]. Er ermöglicht eine Zuordnung der Form und ein gezieltes diagnostisches Vorgehen, wurde mehrfach überarbeitet und unterscheidet aktuell 5 Kategorien: Lymphödeme, die mit vaskulären beziehungsweise kutanen Malformationen und segmentalen Wachstumsstörungen einhergehen, und 4 weitere Kategorien des sogenannten typischen primären Lymphödems [5, 10, 11] ( $\mathbf{A b b . 1 ) .}$

- Tab. 1 Darstellung der bekannten mit einem primären Lymphödem assoziierten Krankheitsbilder inklusive der assoziierten genetischen Veränderungen und der entsprechenden diagnostischen Verfahren. Genauere Informationen zu den Krankheitsbildern finden sich mithilfe der OMIM-Nummer unter www.omim.org. 


\section{Diagnostikalgorithmus}

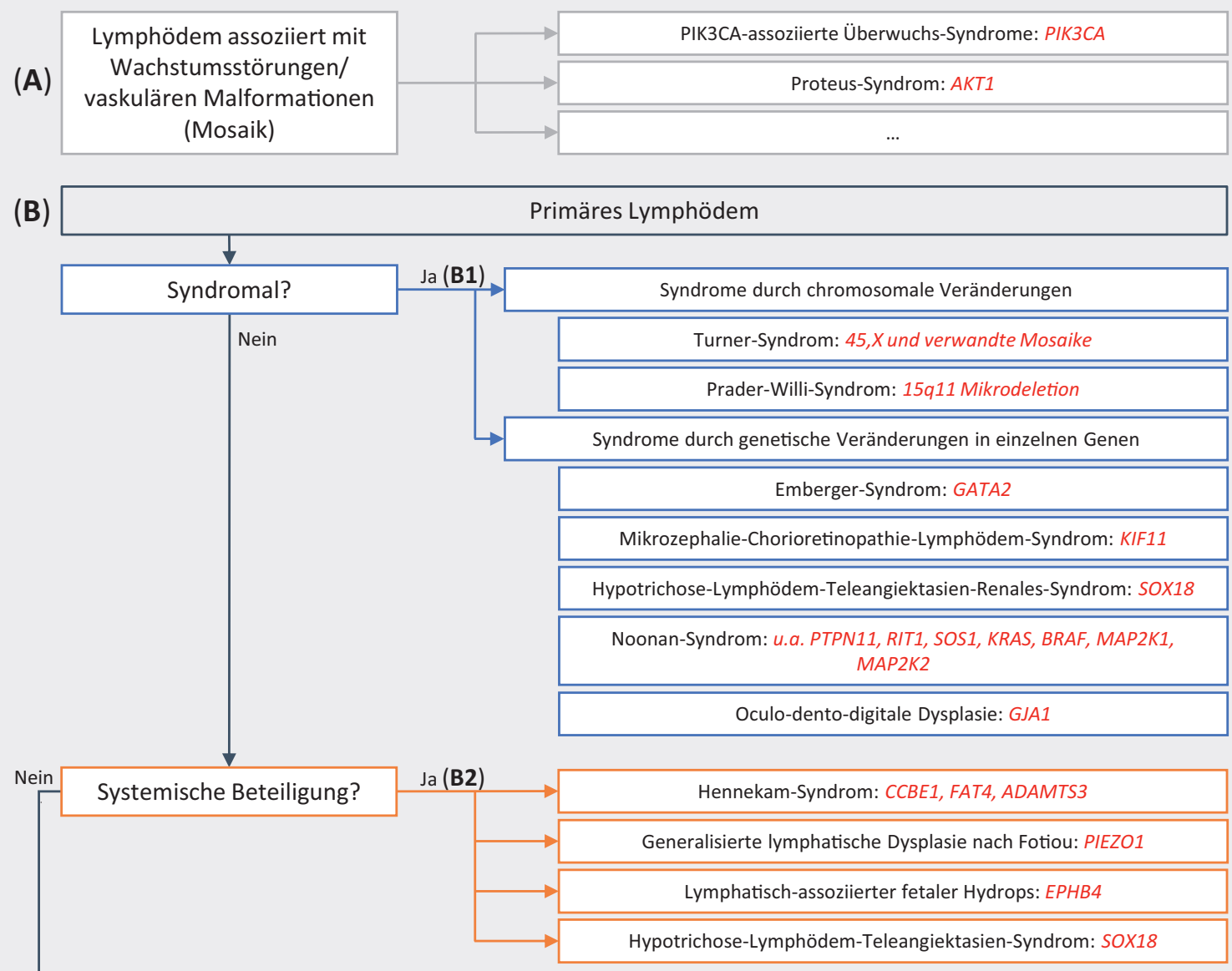

(B3) Kongenitaler Beginn (<1 Jahr alt)

Mongenitales Lymphödem unterschiedlicher Ausprägung ohne
Veränderung in einem bekannten, mit dem Lymphödem assoziierten Gen

(B4) Late-Onset Beginn (>1 Jahr alt)

\begin{tabular}{|c|c|c|}
\hline $\begin{array}{l}\text { Dur Untere } \\
\text { Extremität? }\end{array}$ \\
\cline { 1 - 2 } Nein
\end{tabular}

- Abb. 1 Diagnostikalgorithmus für die Einteilung des primären Lymphödems in 5 farbkodierte Kategorien. Es wird unterschieden zwischen (A) Krankheitsbildern, die auf einem somatischen Mosaik beruhen und mit segmentalen Störungen des Wachstums bzw. Beteiligungen des vaskulären Systems einhergehen (grau), sowie (B) Krankheitsbildern, die mit einem typischen primären Lymphödem einhergehen. Diese bilden wiederum 4 weitere Kategorien: Lymphödeme als Teil einer syndromalen Erkrankung (B1, blau), Krankheitsbilder mit systemischen Beteiligungen (B2, rot), kongenitale Krankheitsbilder, die vor der Vollendung des ersten Lebensjahres auftreten (B3, grün), und Krankheitsbilder, die nach der Vollendung des ersten Lebensjahres auftreten (B4, gelb). Assoziierte genetische Veränderungen bzw. Gene sind rot markiert [11]. 


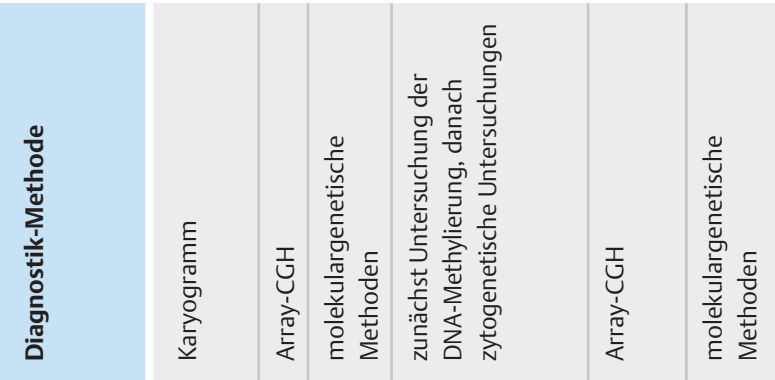

ค

r $6 \times x \times x \times x$
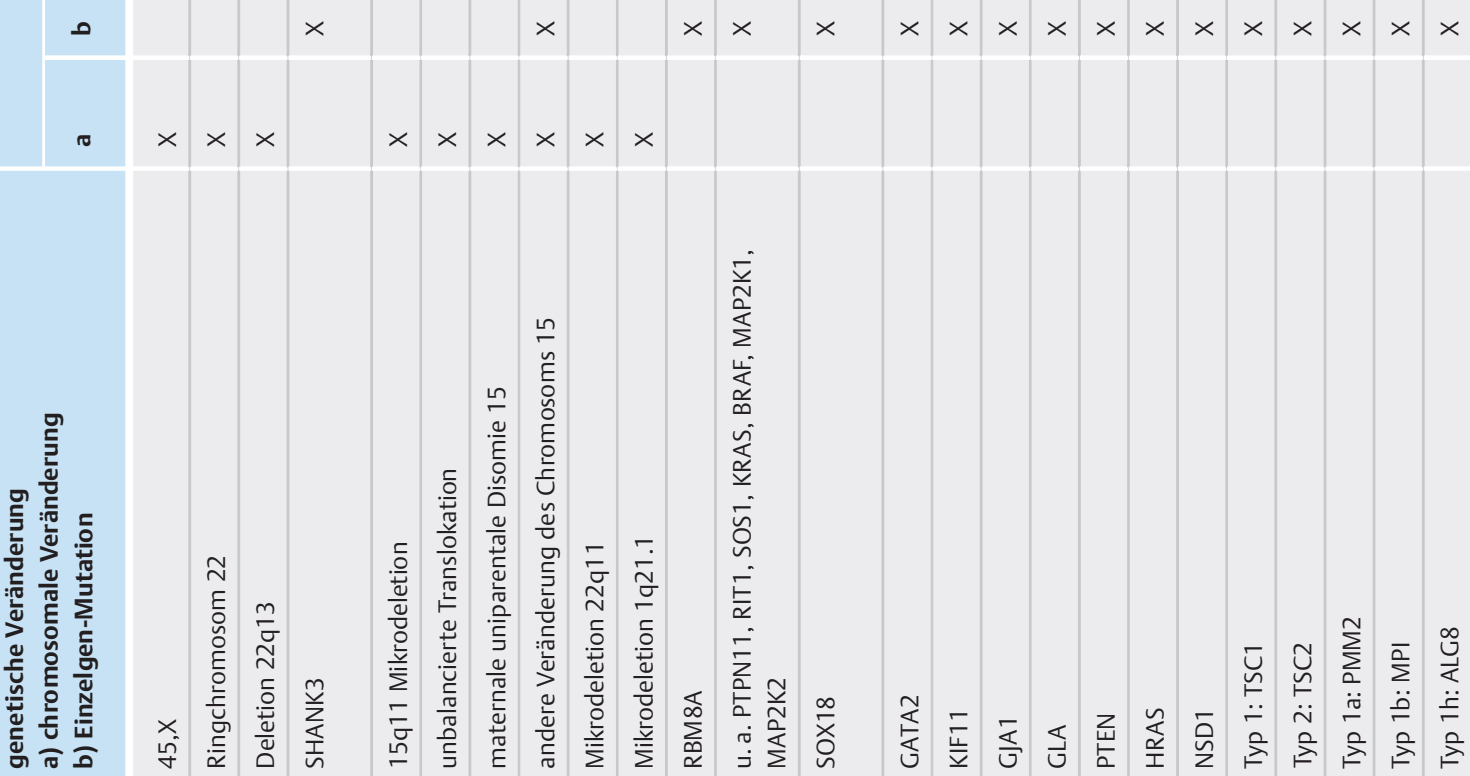

$$
\times \times \times \times \times \times
$$

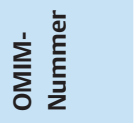

ָ̃

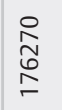

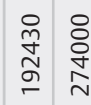

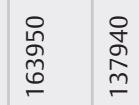






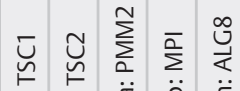

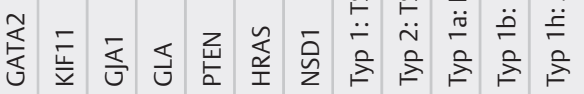
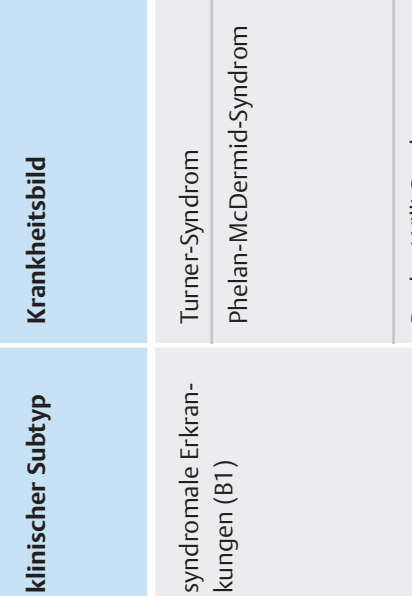







\subsection{Kategorie A: Lymphödem assoziiert mit vaskulä- ren und kutanen Malformationen sowie segmentalen Wachstumsstörungen}

Meist liegt den Krankheitsbildern, die mit segmentalen Wachstumsstörungen und vaskulären Malformationen assoziiert sind, ein somatisches Mosaik zugrunde. Dabei liegt die ursächliche genetische Veränderung nur in einem Teil der Körperzellen vor, weil sie nicht von Anfang an vorhanden war, sondern zu einem späteren postzygotischen Zeitpunkt während der Embryogenese aufgetreten ist. Dementsprechend ist nur der Bereich des Körpers betroffen, der aus dieser ursprünglich mutierten Zelle hervorgegangen ist oder in ihrem Einflussbereich liegt [12]. Die assoziierten Gene besitzen überwiegend eine anti-apoptotische Funktion. Somit begünstigt die pathogene Mutation ein übermäßiges Wachstum der betroffenen Gewebe, wobei neben dem lymphatischen System auch Blutgefäße, Bindegewebe, Fettgewebe und Knochen betroffen sein können [5].

Aktivierende Mutationen im PIK3CA-Gen und im AKT1-Gen führen durch eine Aktivierung des PI3K-AKT-mTOR-Signalwegs zu einer Steigerung der Proliferation, des Zellwachstums und des Zellüberlebens. Bei den PIK3CA-assoziierten Überwuchs-Syndromen (CLOVE-Syndrom, OMIM \#612 918) betrifft die zugrunde liegende somatische Mutation das PIK3CA-Gen, das für die katalytische Untereinheit der Phosphatidylinositol-3-Kinase (PI3K) kodiert [13]. Aktivierende somatische Mutationen im AKT1-Gen verursachen das Proteus-Syndrom (OMIM \#176 920), ein hochvariables Krankheitsbild mit asymmetrischem und disproportionalem Überwuchs von Körperteilen, dysreguliertem Fettgewebe und vaskulären Fehlbildungen. Viele dieser Merkmale finden sich auch bei anderen Überwuchssyndromen [5, 14].

\subsection{Kategorie B: Primäres Lymphödem}

Ursächlich für das primäre Lymphödem sind dagegen Keimbahnmutationen. Diese sind entweder ererbt oder bei Betroffenen neu (de novo) entstanden, betreffen jedoch stets sämtliche Körperzellen. Die assoziierten Gene spielen eine wichtige Rolle in der Lymphangiogenese. Ist diese gestört, kommt es durch Fehlbildung oder Fehlfunktion der Lymphgefäße oder -klappen zum Lymphödem [3]. Entsprechende Krankheitsbilder lassen sich einer der verbliebenen 4 Kategorien zuteilen [11]. Zur ersten Kategorie des primären Lymphödems, den syndromalen Erkrankungen, zählen vorrangig Erkrankungen, bei denen das primäre Lymphödem zwar typischerweise auftritt, aber nicht dominierendes Merkmal ist [10]. Im Gegensatz dazu werden Syndrome, bei denen das primäre Lymphödem im Vordergrund steht, einer der übrigen 3 Kategorien zugeteilt [5].

\subsubsection{Kategorie B1: Syndromale Erkrankungen}

Ursächlich für genetisch bedingte Syndrome sind größere strukturelle Veränderungen der Chromosomen oder Veränderungen in einzelnen Genen. Das Turner-Syndrom geht typischerweise mit einem primären Lymphödem einher und wird durch den durchgängigen Karyotyp 45,X, aber auch verwandte gonosomale Mosaike wie $45, X / 46, X X$ oder $45, X / 46, X Y$ verursacht, bei denen in den betroffenen Zellen ein Geschlechtschromosom fehlt und nur noch ein X-Chromosom vorliegt. Betroffene entwickeln einen weiblichen Phänotyp. Viele Schwangerschaften mit dieser chromosomalen Aberration gehen durch einen schwerwiegenden Hydrops fetalis bereits vor der Geburt verloren. Auch bei Lebendgeborenen mit Turner-Syndrom kann in vielen Fällen pränatal ein Hydrops fetalis diagnostiziert werden, der sich jedoch weitestgehend zurückbildet, sodass postnatal oftmals nur noch ein Pterygium colli und ein primäres Lymphödem der Hand- und Fußrücken zu sehen sind. Weitere Auffälligkeiten beim Turner-Syndrom sind unter anderem angeborene Herzfehler, Kleinwuchs und Gonadendysgenesie. Die Bestimmung des zugrunde liegenden Karyotyps ist wichtig, da Mosaike mit einer 46,XY-Zelllinie mit einem Risiko für maligne Keimzelltumoren assoziiert sind. Klinisch kann das Turner-Syndrom variabel ausgeprägt sein, wobei das primäre Lymphödem typischerweise lediglich ein Teilsymptom darstellt $[15,16]$.

$\mathrm{Zu}$ den auf Genmutationen beruhenden monogenen Syndromen, die mit einem primären Lymphödem assoziiert sind, zählen insbesondere das Emberger-Syndrom (OMIM \#614038), das Noonan-Syndrom (OMIM \#163950), das Mikrozephalie-Chorioretinopathie-Lymphödem-Syndrom (OMIM \#152 950) und die okulo-dento-digitale Dysplasie (OMIM \#164200).

Ursächliche Mutationen für das Emberger-Syndrom finden sich im GATA2-Gen. Dieses kodiert für einen Transkriptionsfaktor, der insbesondere in hämatopoetischen Stammzellen und Endothelzellen exprimiert wird. Klinisch kann die Kombination eines spätmanifesten primären Lymphödems der unteren Extremitäten und Genitalien mit einer Myelodysplasie, die sich zu einer akuten myeloischen Leukämie (AML) weiterentwickeln kann, beobachtet werden [17]. Daher ist bei Betroffenen mit Emberger-Syndrom eine engmaschige Kontrolle zur Früherkennung der AML erforderlich, und das GATA2-Gen sollte grundsätzlich bei primärem Lymphödem differenzialdiagnostisch mituntersucht werden.

Neben dem primären Lymphödem zählen zu den klinischen Zeichen des Noonan-Syndroms postnataler Kleinwuchs, charakteristische faziale Dysmorphie, angeborene Herzfehler, Fehlbildungen des Urogenitaltrakts und manchmal auch Entwicklungsverzögerungen $[18,19]$. Je nach assoziiertem Gen wird das molekulargenetisch bestätigte Noonan-Syndrom mit verschiedenen Typen bezeichnet, gehört aber wie das CFC-Syndrom (OMIM \#115 150) und das Costello-Syndrom (OMIM \#218 040) zu den sogenannten RASopathie-Syndromen. Allen gemeinsam ist, dass sich ursächliche Mutationen in einem der Gene befinden, die für Proteine der RAS-Signalkaskade kodieren. Dieser Signalweg vermittelt die Aktivierung der Zellproliferation, des Zellüberlebens und der Zelldifferenzierung nach der Bindung extrazellulärer Liganden wie Wachstumshormone oder Zytokine [18]. Die Signaltransduktion kann durch Mutationen in einem der Gene PTPN11, RIT1, SOS1, KRAS, BRAF, MAP2K1, MAP2K2 gestört sein, wobei etwa $50 \%$ der nachweisbaren ursächlichen Mutationen das PTPN11-Gen betreffen [5, 18].

Das Mikrozephalie-Chorioretinopathie-Lymphödem-Syndrom ist charakterisiert durch Fehlbildungen des Gehirns, der Augen und des lymphatischen Systems. Im Besonderen ist es meistens assoziiert mit einer milden bis moderaten Intelligenzminderung, einer Chorioretinopathie und einem primären Lymphödem der unteren Extremität, im Besonderen der Fußrücken. Auftreten und Kombination der Merkmale sind variabel. Ursächliche Muta- 
tionen stören die Mitose und den Transport von Vesikeln und finden sich im KIF11-Gen, das ein Motorprotein kodiert [5].

Die okulo-dento-digitale Dysplasie ist mit einem primären Lymphödem assoziiert, betrifft aber insbesondere die Augen (okulo) mit Mikrophthalmie und anderen Augenanomalien, die Zähne (dento) mit kleinen oder fehlenden Zähnen sowie schwachem Zahnschmelz und die Finger (digital) mit einer Kamptodaktylie oder einer Syndaktylie zwischen dem vierten und fünften Finger, manchmal auch der Zehen [20]. Ursächlich sind Mutationen im GJA1-Gen, das für das Protein Connexin 43 kodiert. Gap Junctions, die von Connexin-43-Proteinen gebildet werden, finden sich in vielen Geweben, unter anderem auch in den Klappen der Lymphgefäße, was diese Assoziation erklärt [5, 21].

\subsubsection{Kategorie B2: Primäres Lymphödem mit systemischer Beteiligung}

Die zweite Kategorie des primären Lymphödems umfasst Krankheitsbilder mit systemischer Beteiligung, bei denen das Lymphödem das Leitsymptom ist. Ein Beispiel hierfür ist das HennekamSyndrom, bei dem es zu einem progredienten, kongenitalen primären Lymphödem kommt, das den gesamten Körper betreffen kann. Systemische Manifestationen wie intestinale Lymphangiektasien, Hydrops fetalis, Chylothorax und Aszites sind häufig. Zudem finden sich spezifische faziale Auffälligkeiten. Ursächlich für das Hennekam-Syndrom sind Mutationen im CCBE1-Gen (Hennekam-Syndrom Typ 1, OMIM \#235510), im FAT4-Gen (Hennekam-Syndrom Typ 2, OMIM \#616006) oder im ADAMTS3-Gen (Hennekam-Syndrom Typ 3, OMIM \#618154) [5]. Mutationen in FAT4 beeinträchtigen die Ausbildung der Lymphgefäßklappen [5]. Im Gegensatz dazu spielen CCBE1 und ADAMTS3 eine Rolle in der Prozessierung des lymphatischen Wachstumsfaktors VEGF-C und somit in der Migration von lymphatischen Endothelzellen (LEC) während der embryonalen Entwicklung. Da VEGF-C der dominierende Wachstumsfaktor ist, der die Lymphangiogenese vorantreibt, beeinflussen CCBE1 und ADAMTS3 durch Modulation der VEGF-C-Aktivität die Migration der LECs und die Proliferation der Lymphgefäße [3, 5, 22]. Auf VEGF-C selbst wird unter 2.2.3 genauer eingegangen, da Mutationen im VEGFC-Gen mit der Milroy-ähnlichen Erkrankung assoziiert sind, die einer anderen Kategorie zugeordnet wird.

Die generalisierte lymphatische Dysplasie nach Fotiou (OMIM \#616843) ähnelt dem Bild eines Hennekam-Syndroms, jedoch ist das primäre Lymphödem geringer ausgeprägt. Ursächlich sind Mutationen im PIEZO1-Gen, das für einen mechanosensitiven lonenkanal kodiert. Dieser beeinflusst die Lymphangiogenese über die Vermittlung mechanischer Reize [23].

Ein weiteres Syndrom, das mit einem Lymphödem mit systemischen Beteiligungen assoziiert ist, ist das Hypotrichose-Lymphödem-Teleangiektasien-Syndrom (HLTS, OMIM \#607823), bei dem das Lymphödem hauptsächlich die untere Extremität betrifft. Die systemischen Beteiligungen umfassen hauptsächlich einen Hydrops fetalis, eine Hydrozele bei männlichen Patienten und Beteiligungen der Haut mit Teleangiektasien. Ursächlich sind Mutationen in SOX18 [5]. SOX18 spielt eine kritische Rolle in der embryonalen Lymphangiogenese, weil es die Expression des lymphatischen Master-Transkriptionsfaktors PROX1 induziert, der wiederum die Differenzierung von LECs aus venösen Blutendothelzellen initiiert [3]. Bei Betroffenen mit Mutationen im SOX18Gen kann es auch zu einer renalen Beteiligung kommen. In diesem Fall lautet die Diagnose Hypotrichose-Lymphödem-Teleangiektasien-Renales-Syndrom (HLTRS, OMIM\#137940). Allerdings wird dieses Syndrom im Gegensatz zum HLTS laut Algorithmus der Kategorie B1 Syndromale Erkrankungen zugeteilt [5].

\subsubsection{Kategorie B3: Kongenitales primäres Lymphödem}

Wenn das primäre Lymphödem weder im Rahmen einer übergeordneten syndromalen Erkrankung auftritt noch mit systemischen Beteiligungen assoziiert ist, erfolgt die weitere Einteilung anhand des Zeitpunkts der ersten klinischen Manifestation. Man unterscheidet zwischen dem kongenitalen und dem spätmanifestierenden (Late-Onset) primären Lymphödem. Das kongenitale primäre Lymphödem tritt bereits pränatal oder innerhalb des ersten Lebensjahres auf $[5,10]$.

Das klassische Krankheitsbild des kongenitalen Lymphödems ist die Milroy-Erkrankung (OMIM \#153100). Sie ist eine der häufigsten Ursachen für ein primäres Lymphödem und zeichnet sich durch ein meist bilaterales Lymphödem der unteren Extremitäten aus, welches vorwiegend unterhalb des Knies und dabei im Besonderen auf dem Fußrücken auftritt. Das Ausmaß des Lymphödems bleibt bei den meisten Betroffenen im Laufe des Lebens konstant. Des Weiteren liegen bei den Betroffenen oft Varikosen am Bein und Fuß vor. Ursächlich für die klassische Milroy-Erkrankung sind Mutationen im FLT4-Gen, das für den vaskulären Wachstumsfaktor-Rezeptor namens Vascular Endothelial Growth Factor Receptor 3 (VEGFR-3) kodiert [24]. Dieser Rezeptor wird von LECs exprimiert und unter anderem durch die Bindung von VEGF-C aktiviert [3]. So erklärt sich, weshalb Mutationen im VEGFC-Gen zur selteneren Milroy-ähnlichen Erkrankung (OMIM \#615907) führen, die sich klinisch wie die klassische Milroy-Erkrankung mit einem kongenitalen Lymphödem der unteren Extremität, im Besonderen unterhalb des Knies, und Varikosen präsentiert [25]. Betroffene mit Milroy- oder Milroy-ähnlicher Erkrankung haben typischerweise eine positive Familienanamnese für kongenitale Lymphödeme. Eine negative Familienanamnese bei bilateralem Ödem der unteren Extremität schließt diese Verdachtsdiagnosen jedoch nicht aus [5].

Darüber hinaus gibt es einige Formen des kongenitalen Lymphödems, bei denen das assoziierte Gen bislang unbekannt ist, wie multisegmentale und unilaterale Ödeme sowie Ödeme mit Beteiligung der Genitalien [5].

\subsubsection{Kategorie B4: Late-Onset-primäres Lymphödem}

Betroffene mit einem Late-Onset-Lymphödem sollten zuerst auf Vorliegen einer Distichiasis untersucht werden. Dabei handelt es sich um eine zweite Reihe von Wimpern oder auch nur einzelne Wimpern, die aus den Meibom 'schen Drüsen auf der Innenseite des Augenlids wachsen. Das Vorliegen einer Distichiasis in Verbindung mit einem meist in der Pubertät auftretenden primären Lymphödem und dem frühen Auftreten von Varikosen ist charakteristisch für das Lymphödem-Distichiasis-Syndrom (OMIM \#153400) [5, 26]. Ursächlich sind Mutationen im FOXC2-Gen. Der kodierte Transkriptionsfaktor reguliert unter anderem die 

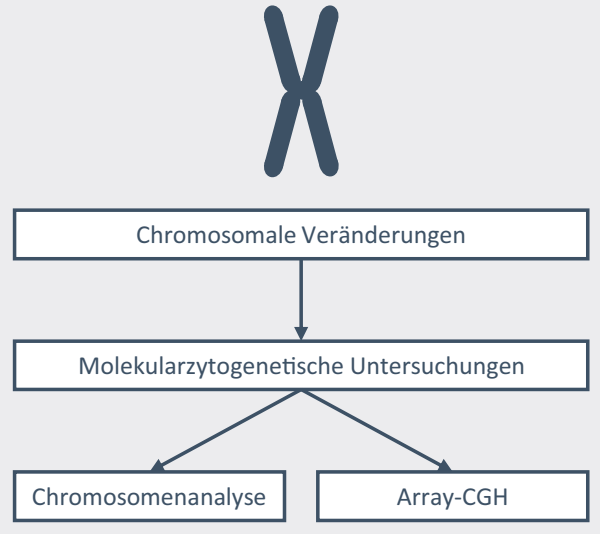
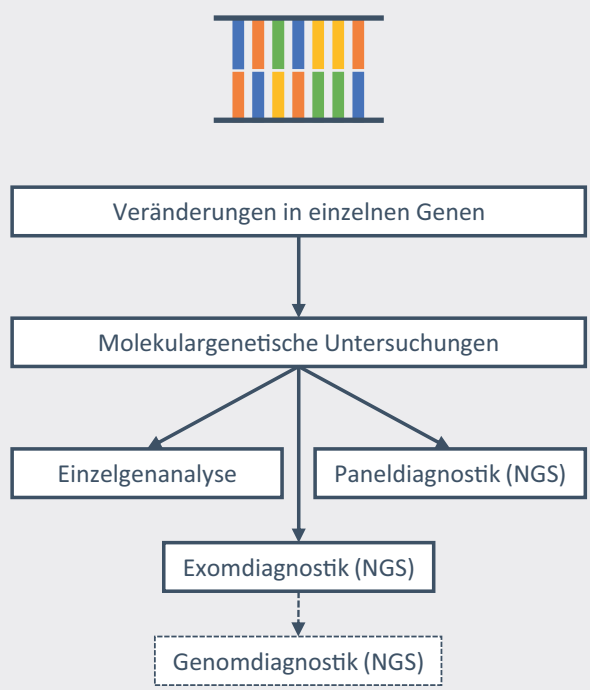

- Abb.2 Übersicht über mögliche diagnostische Verfahren in der Humangenetik bei Verdacht auf Vorliegen eines primären Lymphödems. Durch zytogenetische Untersuchungen können chromosomale Veränderungen wie Inversionen, Deletionen und Translokationen (Chromosomenanalyse) sowie Mikrodeletionen und Mikroduplikationen (Array-CGH) festgestellt werden. Mit den molekulargenetischen Untersuchungen können Veränderungen in einzelnen Genen untersucht werden - entweder mittels Einzelgenanalyse oder mittels Next Generation Sequencing (NGS), beispielsweise im Rahmen einer Paneldiagnostik, Exomdiagnostik oder Genomdiagnostik (vorwiegend für Forschungszwecke).

Entwicklung der Klappen in Lymphgefäßen und Venen. Bei Fehlbildung dieser kommt es über einen gestörten Abtransport der Flüssigkeit mit Reflux zur Ausbildung des Lymphödems und der Varikosen [3, 5].

Wie das Lymphödem-Distichiasis-Syndrom gehört die MeigeErkrankung (OMIM \#153200) zu den häufigsten Krankheitsbildern mit einem primären Lymphödem. Sie geht ebenfalls mit einem Lymphödem der unteren Extremität einher, das sich erst in der Adoleszenz oder im Erwachsenenalter ausprägt, unterscheidet sich jedoch durch das isolierte Auftreten des Lymphödems ohne Varikosis. Zwar zeigt die Meige-Erkrankung eine familiäre Häufung, bisher konnte jedoch kein assoziiertes Gen identifiziert werden $[10,11]$.

Beim Late-Onset-Lymphödem ist die untere Extremität am häufigsten betroffen. Allerdings gibt es auch Krankheitsbilder, bei denen das Lymphödem im Bereich der oberen Extremität oder in der Genitalregion auftreten kann. Ein Beispiel hierfür ist das 4-Extremitäten-Lymphödem (OMIM \#613480), das ebenfalls überwiegend in der Adoleszenz beginnt. Meist sind zunächst die Beine betroffen. Dann breitet sich das progrediente Lymphödem auch auf die Arme aus, die jedoch üblicherweise weniger schwer betroffen sind. Ursächlich sind Mutationen im GJC2-Gen, welches für Connexin 47 kodiert. Gap Junctions aus Connexin-47-Proteinen werden wie Gap Junctions aus Connexin 43 in den Lymphgefäßklappen etabliert. Beide sind für die Signaltransduktion zwischen den Klappen-Endothelzellen und somit für die korrekte Ausbildung der Klappen essenziell. Daher ist sowohl bei der GJA1-assoziierten okulo-dento-digitalen Dysplasie als auch beim GJC2-assoziierten 4-ExtremitätenLymphödem davon auszugehen, dass eine Fehlbildung und Funktionsstörung der Klappen besteht [5].

\section{Genetische Diagnostik}

Konnte ein Patient aufgrund seiner Klinik einer der 5 Kategorien zugeordnet werden, erfolgt die Suche nach der zugrunde liegenden genetischen Veränderung im Rahmen einer Stufendiagnostik. Grundsätzlich sind in der genetischen Diagnostik die Methoden der konventionellen Zytogenetik (Chromosomenanalyse) von den molekularzytogenetischen Methoden wie Array-CGH (Arraybasierte Comparative Genom Hybridisierung) und FISH (Fluoreszenz-in-situ-Hybridisierung) und der Molekulargenetik (Sequenzanalyse, MLPA: für Englisch Multiplex Ligation-dependent Probe Amplification) zu unterscheiden. Bei der postnatalen Suche nach Veränderungen der Keimbahn, die sämtliche Körperzellen betreffen, wird typischerweise das Blut als Stellvertreterorgan untersucht. Dies betrifft somit Erkrankungen der Kategorie B, während die Ursache bei Erkrankungen der Kategorie A, denen somatische Mutationen zugrunde liegen, nur anhand einer Untersuchung im betroffenen Gewebe geklärt werden kann ( $\triangleright$ Abb. 2).

\subsection{Chromosomenanalyse und Array-CGH}

Bei der konventionellen zytogenetischen Diagnostik, also der klassischen Chromosomenanalyse, handelt es sich um eine lichtmikroskopische Analyse der Metaphase-Chromosomen aus Lymphozyten. Für die Chromosomenanalyse können nur lebendige Zellen verwendet werden, die zunächst kultiviert werden, um dann die Zellteilung in der Metaphase anzuhalten und die kondensierten Chromosomen zu bändern. Geeignetes Material für diese Untersuchung sind daher ca. 5-10 ml heparinisiertes Vollblut. Eine pränatale Diagnostik aus Amnion-, Chorionzotten- oder fetalen Blutzellen ist ebenfalls möglich. 
Anhand der Analyse von meist 20 Metaphasen können numerische (Aneuploidien) und strukturelle Veränderungen der Chromosomen wie Inversionen, Deletionen und Translokationen entdeckt werden. Der erhobene Befund wird sodann im Karyotyp zusammengefasst. Der unauffällige Chromosomensatz des Menschen umfasst 46 Chromosomen: 22 Autosomenpaare (geschlechtsunabhängige Chromosomen) und 2 Geschlechtschromosomen. Somit beschreibt der Karyotyp 46,XX einen unauffälligen weiblichen und der Karyotyp 46,XY einen unauffälligen männlichen Chromosomensatz. Mit dieser Untersuchung sind nur lichtmikroskopisch sichtbare Veränderungen an den Chromosomen erfasst.

Kleinere strukturelle Aberrationen (Mikrodeletionen und Mikroduplikationen) können zwar gezielt durch eine FISH auf den präparierten Metaphasen oder in Interphase-Kernen abgeklärt werden, lassen sich aber mit der höher auflösenden Array-CGH genomweit darstellen. Hierfür wird eine EDTA-Blutprobe $(2-5 \mathrm{ml})$ des Patienten benötigt. Der Ausschluss gewebespezifischer oder schwacher Mosaike ist aus methodischen Gründen prinzipiell nicht möglich. Veränderungen an einzelnen Genen (Genmutationen) oder Kopie-neutrale Veränderungen sind mit dieser Methode nicht nachweisbar. Sofern keine konkrete Verdachtsdiagnose vorliegt, stellen die Chromosomenanalyse und Array-CGH in der Regel die ersten Schritte der genetischen Diagnostik bei unklaren syndromalen Erkrankungen dar.

\subsection{Molekulargenetische Diagnostik}

Je nach Umfang der molekulargenetischen Diagnostik, für die ebenfalls EDTA-Blut verwendet wird, unterscheiden wir zwischen einer oder mehreren Einzelgenanalysen oder der parallelen Sequenzierung mehrerer Gene (Multi-Gen-Analyse, Paneldiagnostik), aller bekannten Protein-kodierenden Gene (Exomsequenzierung) und der Gesamtgenomsequenzierung. Anhand der Untersuchung der Gensequenzen können Punktmutationen, also Veränderungen einzelner oder weniger benachbarter Basenpaare der DNA, detektiert werden.

Bei starkem klinischem Verdacht auf eine bestimmte Erkrankung (z. B. bei Vorliegen charakteristischer klinischen Manifestation eines Distichiasis-Lymphödem-Syndroms) kann gezielt das assoziierte Gen im Rahmen einer Einzelgen-Analyse sequenziert werden (in diesem Fall das FOXC2-Gen). Ergänzend können Kopienzahlveränderungen in einzelnen Genen mithilfe der MLPA abgeklärt werden.

Mittels der inzwischen weit verbreiteten Hochdurchsatz-Sequenzierungstechnik (Next Generation Sequencing, NGS) können mehrere Gene parallel sequenziert werden (Multi-Gen-Analyse, Paneldiagnostik). Die NGS-basierten Panelanalysen können wie die Einzelgen-Diagnostik im Rahmen der Regeldiagnostik veranlasst werden und sind kassenärztliche Leistungen. Die umfangreichste Paneldiagnostik stellt die sogenannte Exomsequenzierung dar, bei der die kodierenden Bereiche und flankierenden Intronbereiche aller bekannten Protein-kodierenden menschlichen Gene erfasst werden. Bis vor kurzem war diese Analyse nur im Rahmen von Forschungsprojekten möglich. Inzwischen kommt sie jedoch auch in der Regelversorgung zum Einsatz, wobei dann aktuell nur bis zu 25kb der erhobenen Sequenzen befundet und abgerechnet werden dürfen. Der große Vorteil der
Exomdiagnostik ist die umfassende Sequenzierung, sodass sie sich insbesondere für unklare syndromale Erkrankungen mit Verdacht auf eine seltene Ursache eignet und dabei dennoch eine schnelle Diagnosestellung ermöglicht. Die Exomsequenzierung bietet zudem die Möglichkeit, neue Kandidatengene zu identifizieren, die mit der bestehenden Symptomatik bislang noch nicht in Verbindung gebracht werden konnten. Insbesondere bei betroffenen Kindern wird aktuell häufig eine sogenannte Trio-Exomsequenzierung durchgeführt, bei der neben dem betroffenen Kind auch beide gesunden Eltern sequenziert werden, um so insbesondere De-novo-Mutationen effizient nachzuweisen oder bei vermutetem autosomal-rezessivem Erbgang das biallelische Vererbungsmuster zu bestätigen. Krankheitsrelevante genetische Veränderungen können jedoch auch in nichtkodierenden Genen und Abschnitten des Genoms lokalisiert sein. Diese Bereiche werden selbst bei der Exomsequenzierung nicht erfasst. Die derzeit umfassendste Stufe der Sequenzanalyse stellt nach aktuellem Wissensstand die Genomsequenzierung dar, die jedoch derzeit nur im Rahmen von Forschungsprojekten angeboten werden kann. Die Herausforderung all dieser molekulargenetischen Methoden und damit auch der größte Zeitaufwand begründet sich jedoch nicht in der Sequenzierung selbst, sondern in der Bewertung der gefundenen Varianten, also der Vielzahl von Abweichungen von der Referenzsequenz, die mit dem Umfang der Diagnostik ansteigt.

\section{Zusammenfassung}

Zusammenfassend ist das primäre Lymphödem ein Symptom verschiedenster Krankheitsbilder unterschiedlicher Schwere und klinischer Präsentation, die auf der Grundlage einer genetischen Veränderung entstehen. Diese Krankheitsbilder lassen sich in 5 Kategorien einteilen, wodurch die Verdachtsdiagnosen je nach Symptomkomplex eingegrenzt werden können und im Folgenden eine zielgerichtete genetische Untersuchung (am besten in einem spezialisierten Zentrum) durchgeführt werden kann.

\section{Interessenkonflikt}

Die Autorinnen/Autoren geben an, dass kein Interessenkonflikt besteht.

\section{Literatur}

[1] Mortimer PS, Rockson SG. New developments in clinical aspects of lymphatic disease. J Clin Invest 2014; 124: 915-921. doi:10.1172/jci71608

[2] Lüllmann-Rauch R, Asan E. Lymphgefäße. In: Lüllmann-Rauch R, Asan E, Hrsg. Taschenlehrbuch Histologie. 6., vollständig überarbeitete Auflage. Aufl: Georg Thieme Verlag; 2019. doi:10.1055/b-006-163361

[3] Alitalo K. The lymphatic vasculature in disease. Nat Med 2011; 17: 1371 1380. doi: $10.1038 / \mathrm{nm} .2545$

[4] Kröger K. Lymphödem. In: Suttorp N, Möckel M, Siegmund B, et al. Hrsg. Harrisons Innere Medizin. 20. Auflage. Aufl ABW Verlag; 2020. doi:10.1055/b000000107

[5] Mansour S, Martin-Almedina S, Ostergaard P. Genetic Disorders of the Lymphatic System. In: Pyeritz RE, Korf BR, Grody WW, Hrsg. Emery and Rimoin's Principles and Practice of Medical Genetics and Genomics 
(Seventh Edition); 2020: 231-249. doi:10.1016/B978-0-12-8125328.00008-2

[6] Jones GE, Mansour S. An approach to familial lymphoedema. Clin Med (Lond) 2017; 17: 552-557. doi:10.7861/clinmedicine.17-6-552

[7] Dempsey E, Homfray T, Simpson JM et al. Fetal hydrops - a review and a clinical approach to identifying the cause. Expert Opinion on Orphan Drugs 2020; 8: 51-66. doi:10.1080/21678707.2020.1719827

[8] Bellini C, Hennekam RC, Bonioli E. A diagnostic flow chart for nonimmune hydrops fetalis. Am J Med Genet A 2009; 149a: 852-853. doi:10.1002/ajmg.a.32677

[9] Bellini C, Hennekam RC. Non-immune hydrops fetalis: a short review of etiology and pathophysiology. Am J Med Genet A 2012; 158a: 597-605. doi:10.1002/ajmg.a.34438

[10] Connell FC, Gordon K, Brice G et al. The classification and diagnostic algorithm for primary lymphatic dysplasia: an update from 2010 to include molecular findings. Clin Genet 2013; 84: 303-314. doi:10.1111/ cge. 12173

[11] Gordon K, Varney R, Keeley V et al. Update and audit of the St George's classification algorithm of primary lymphatic anomalies: a clinical and molecular approach to diagnosis. J Med Genet 2020; 57: 653-659. doi:10.1136/jmedgenet-2019-106084

[12] Zschocke J. Pathomechanismen genetischer Krankheiten. In Basiswissen Humangenetik. Berlin, Heidelberg: Springer Berlin Heidelberg; 2018: 67-95. doi:10.1007/978-3-662-56147-8_4

[13] Kurek KC, Luks VL, Ayturk UM et al. Somatic mosaic activating mutations in PIK3CA cause CLOVES syndrome. Am J Hum Genet 2012; 90: 11081115. doi:10.1016/j.ajhg.2012.05.006

[14] Cohen MM Jr. Proteus syndrome review: molecular, clinical, and pathologic features. Clin Genet 2014; 85: 111-119. doi:10.1111/cge.12266

[15] Morgan T. Turner syndrome: diagnosis and management. Am Fam Physician 2007; 76: 405-410

[16] Schaaf CP. Angeborene Fehlbildungssyndrome. In Basiswissen Humangenetik. Berlin, Heidelberg: Springer Berlin Heidelberg; 2018: 241-262. doi:10.1007/978-3-662-56147-8_19

[17] Ostergaard P, Simpson MA, Connell FC et al. Mutations in GATA2 cause primary lymphedema associated with a predisposition to acute myeloid leukemia (Emberger syndrome). Nat Genet 2011; 43: 929-931. doi:10.1038/ng.923

[18] Roberts AE, Allanson JE, Tartaglia M et al. Noonan syndrome. Lancet 2013; 381: 333-342. doi:10.1016/s0140-6736(12)61023-x

[19] Schaaf CP. Kreislaufsystem und Hämatologie. In Basiswissen Humangenetik. Berlin, Heidelberg: Springer Berlin Heidelberg; 2018: 275-294. doi:10.1007/978-3-662-56147-8_21

[20] Brice G, Ostergaard P, Jeffery $S$ et al. A novel mutation in GJA1 causing oculodentodigital syndrome and primary lymphoedema in a three generation family. Clinical Genetics 2013; 84: 378-381. doi:10.1111/ cge. 12158

[21] Paznekas WA, Karczeski B, Vermeer S et al. GJA1 mutations, variants, and connexin 43 dysfunction as it relates to the oculodentodigital dysplasia phenotype. Human Mutation 2009; 30: 724-733. doi:10.1002/ humu.20958

[22] Hägerling R, Pollmann C, Andreas M et al. A novel multistep mechanism for initial lymphangiogenesis in mouse embryos based on ultramicroscopy. Embo J 2013; 32: 629-644. doi:10.1038/emboj.2012.340

[23] Fotiou E, Martin-Almedina S, Simpson MA et al. Novel mutations in PIEZO1 cause an autosomal recessive generalized lymphatic dysplasia with non-immune hydrops fetalis. Nat Commun 2015; 6: 8085. doi:10.1038/ ncomms9085

[24] Brice G, Child AH, Evans A et al. Milroy disease and the VEGFR-3 mutation phenotype. J Med Genet 2005; 42: 98-102. doi:10.1136/ jmg.2004.024802

[25] Gordon K, Schulte D, Brice G et al. Mutation in vascular endothelial growth factor-C, a ligand for vascular endothelial growth factor receptor-3, is associated with autosomal dominant milroy-like primary lymphedema. Circ Res 2013; 112: 956-960. doi:10.1161/circresaha. 113.300350

[26] Mansour S, Brice GW, Jeffery S et al. Lymphedema-Distichiasis Syndrome. In: Adam MP, Ardinger HH, Pagon RA et al., Hrsg. GeneReviews (®). Seattle (WA): University of Washington, Seattle. Copyright @ $1993-$ 2020, University of Washington, Seattle. GeneReviews is a registered trademark of the University of Washington, Seattle. All rights reserved 2005 (updated 2019) 